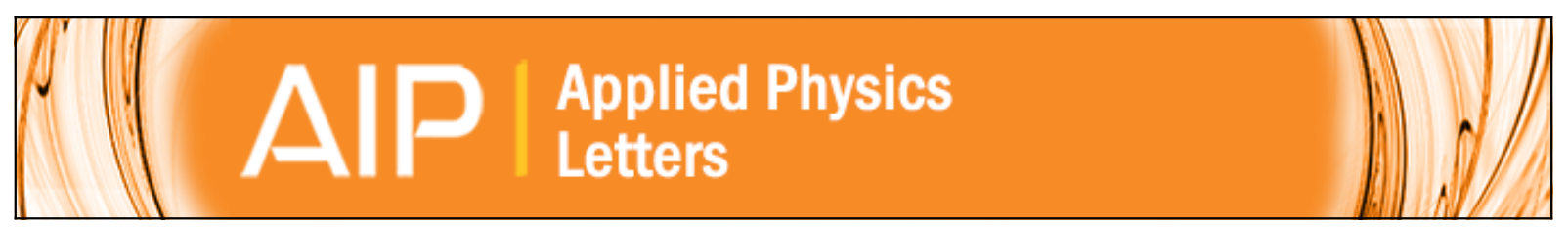

\title{
Type II InAs/GaAsSb quantum dots: Highly tunable exciton geometry and topology
}

J. M. Llorens, L. Wewior, E. R. Cardozo de Oliveira, J. M. Ulloa, A. D. Utrilla, A. Guzmán, A. Hierro, and B. Alén

Citation: Applied Physics Letters 107, 183101 (2015); doi: 10.1063/1.4934841

View online: http://dx.doi.org/10.1063/1.4934841

View Table of Contents: http://scitation.aip.org/content/aip/journal/apl/107/18?ver=pdfcov

Published by the AIP Publishing

\section{Articles you may be interested in}

Excitonic transitions in highly efficient (Galn)As/Ga(AsSb) type-Il quantum-well structures

Appl. Phys. Lett. 107, 182104 (2015); 10.1063/1.4935212

Highly tensile-strained, type-II, Ga 1 - x In x As / GaSb quantum wells

Appl. Phys. Lett. 96, 062109 (2010); 10.1063/1.3303821

GaAs $0.7 \mathrm{Sb} 0.3$ / GaAs type-II quantum well with an adjacent InAs quantum-dot stressor layer Appl. Phys. Lett. 94, 111106 (2009); 10.1063/1.3100191

Effects of thermal annealing on the emission properties of type-II InAs/GaAsSb quantum dots Appl. Phys. Lett. 94, 053101 (2009); 10.1063/1.3062979

A comparative study of spontaneous emission and carrier recombination processes in InGaAs quantum dots and GalnNAs quantum wells emitting near $1300 \mathrm{~nm}$

J. Appl. Phys. 92, 6215 (2002); 10.1063/1.1512683

\section{AIP $\mid$ APL Photonics}

APL Photonics is pleased to announce Benjamin Eggleton as its Editor-in-Chief

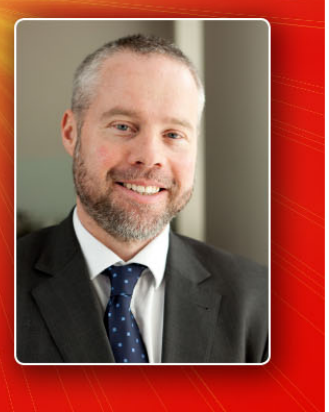




\title{
Type II InAs/GaAsSb quantum dots: Highly tunable exciton geometry and topology
}

\author{
J. M. Llorens, ${ }^{1}$ L. Wewior, ${ }^{1}$ E. R. Cardozo de Oliveira, ${ }^{1, a)}$ J. M. Ulloa, ${ }^{2}$ A. D. Utrilla, ${ }^{2}$ \\ A. Guzmán, ${ }^{2}$ A. Hierro, ${ }^{2}$ and B. Alén ${ }^{1, b)}$ \\ ${ }^{1}$ IMM-Instituto de Microelectrónica de Madrid (CNM-CSIC), Isaac Newton 8, PTM, E-28760 Tres Cantos, \\ Madrid, Spain \\ ${ }^{2}$ Institute for Systems based on Optoelectronics and Microtechnology (ISOM), Universidad Politécnica \\ de Madrid, Ciudad Universitaria s/n, 28040 Madrid, Spain
}

(Received 20 July 2015; accepted 19 October 2015; published online 2 November 2015)

\begin{abstract}
External control over the electron and hole wavefunctions geometry and topology is investigated in a p-i-n diode embedding a dot-in-a-well InAs/GaAsSb quantum structure with type II band alignment. We find highly tunable exciton dipole moments and largely decoupled exciton recombination and ionization dynamics. We also predicted a bias regime where the hole wavefunction topology changes continuously from quantum dot-like to quantum ring-like as a function of the external bias. All these properties have great potential in advanced electro-optical applications and in the investigation of fundamental spin-orbit phenomena. (C) 2015 AIP Publishing LLC.
\end{abstract}

[http://dx.doi.org/10.1063/1.4934841]

Semiconductor quantum dots (QDs) play a key role in ultrafast signal processing devices such as electro-optical modulators, switches, and delay generators. ${ }^{1-3}$ Made with different semiconductor materials, such as GaAs, InP, Si/Ge, etc., semiconductor based devices work stable at ultrahigh speeds using a fraction of the energy and space needed by competing electro-optical materials. ${ }^{4}$ Band-gap engineered semiconductors, and QDs and quantum wells (QWs), in particular, have made this possible with great success, thanks to a precise control of electronic excitations via an externally applied electric (or electromagnetic) field; a key concept which, beyond the modulation of light phase and amplitude, underpins many other cutting-edge quantum technologies from excitonic integrated circuits to quantum memories. ${ }^{5-8}$

For many years, the prototypical system to develop new classical and quantum information technologies has been self-assembled In(Ga)As/GaAs QDs. Owing to its type I band alignment, electrons and holes in this system are strongly confined within the $\operatorname{In}(\mathrm{Ga})$ As material, being the response of their ground state wavefunctions to the external bias correspondingly small and similar for both carriers. ${ }^{9-11}$ However, for type II band alignment, only the hole or the electron is confined within the QD, whereas the companion particle remains weakly localized outside, and the electrooptical response is comparatively unexplored. It is expected that the weakly confined particle would be more sensitive to external stimuli, resulting in a larger electrical polarizability along with other characteristics associated to the very different confinement regime for electrons and holes. ${ }^{12,13}$ All these features might vary between type I and type II QDs and deserve a careful investigation.

To explore these fundamental differences, we have embedded an InAs/GaAsSb dot-in-a-well structure in a GaAs

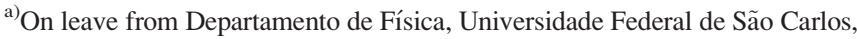
13.565-905 São Carlos, São Paulo, Brazil.

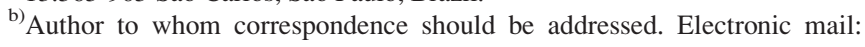
benito.alen@csic.es
}

$\mathrm{p}-\mathrm{i}-\mathrm{n}$ diode and investigated its optical properties as a function of the external electric field at $5 \mathrm{~K}$. Figure 1(a) shows a schematic representation of the device under study. More details about the sample growth, device fabrication, and characterization along with a description of the $8 \times 8 \mathbf{k} \cdot \mathbf{p}$ model which support our findings can be found in the supplementary material. ${ }^{14}$

Traditionally, $\mathrm{Sb}$ has been employed either within the InAs QDs ${ }^{15,16}$ or in the capping ${ }^{17,18}$ to shift their emission wavelength into the $\mathrm{O}$ and $\mathrm{C}$ optical telecommunication bands. Depending on the amount of $\mathrm{Sb}$, this redshift comes along with a transition from type I to type II band alignment at the QD interfaces. ${ }^{19-22}$ In this study, for the particular amount of $\mathrm{Sb}$ used in the cap layer, the valence band alignment across the GaAsSb/InAs interfaces turns from type I to type II, leaving the electrons confined within the InAs QD,
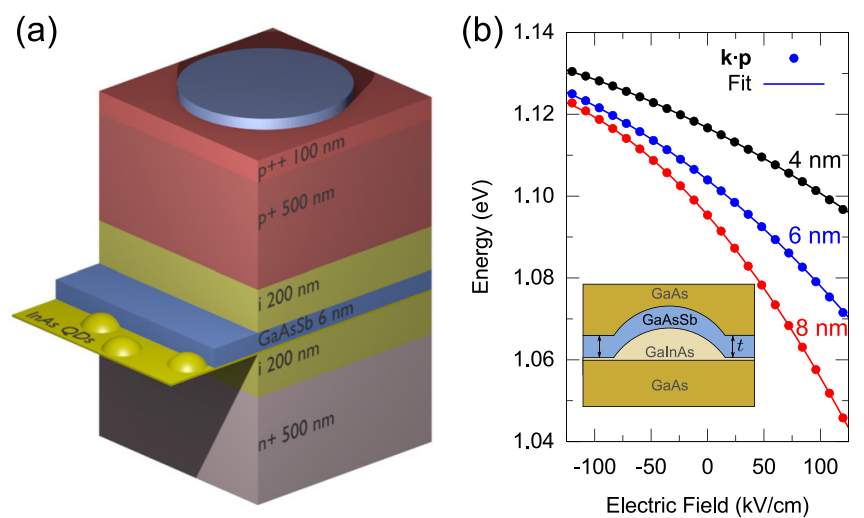

FIG. 1. (a) Schematic representation of the investigated device structure with doping concentrations, layer thicknesses, and active layer composition indicated. (b) Symbols are k·p calculations of the ground state energy dispersion in an external electric field for different thicknesses $(t)$ of the GaAsSb QW. Black, blue, and red solid lines fit to the expression $E(F)=E_{0}-p F+\beta F^{2}$ using $p / e(\mathrm{~nm})=1.4,2.2$, and 3.2 and $\beta\left(\mu \mathrm{eV} \mathrm{kV} \mathrm{km}^{2}\right)=-0.21,-0.39$, and -0.78 , respectively. The inset shows a detailed scheme of the active layer composition and geometry used for the calculation. 
while expelling the holes to the capping layer already at zero electric field.

Besides type II band alignment, the vicinity of the top $\mathrm{GaAsSb} / \mathrm{GaAs}$ interface, located a few nanometers above the QD apex, is a key element in our design (see the inset in Figure 1(b)). First, it acts as an effective barrier for the holes, preventing the weakly localized hole to drift away and ionize from the electron Coulomb potential. ${ }^{23}$ Second, it allows a precise control of the key parameters that govern the response to the external field in electro-optical applications. Under these conditions, we find a bias regime where the hole wavefunction winds around the electron one, inducing a large in-plane dipole moment. This comes along with a change in the exciton topology from singly connected to doubly connected, that might have a strong impact on voltage tunable spin-orbit physics and spintronic devices beyond the electro-optical realm. ${ }^{24}$

The electronic properties of an electron-hole system spatially confined in the direction of an external electric field, $F$, are governed by the quantum confined Stark-effect (QCSE). ${ }^{25}$ Its impact on the ground state energy can be described up-to second order in the perturbation theory by the quadratic function $E(F)=E_{0}-p F+\beta F^{2}$, where the parameter $p$ represents the permanent vertical dipole moment and $\beta$ the polarizability. Figure 1(b) shows that, despite the complex confinement geometry, this simple parabolic function accurately describes the ground state energy evolution calculated using three dimensional eight-band $\mathbf{k} \cdot \mathbf{p}$ methods as implemented in the Nextnano++ software package. ${ }^{26}$ It is important to highlight that the value of $p$ and $\beta$ can be changed only by increasing or reducing the GaAsSb QW thickness, $t$. This brings a great advantage over type I systems, where such manipulation relies on changing the dimensions of the QD, which are more difficult to access experimentally in self-assembled nanostructures.

Figures 2 and 3 show a direct comparison between the calculated and experimental data. The evolution of the photoluminescence (PL) spectrum measured at $5 \mathrm{~K}$ as a function of the device bias is displayed in Figure 2(a). ${ }^{27}$ At $0 \mathrm{~V}$, the spectrum is dominated by the ground state emission centered at $1.134 \mathrm{eV}$ with full width at half maximum

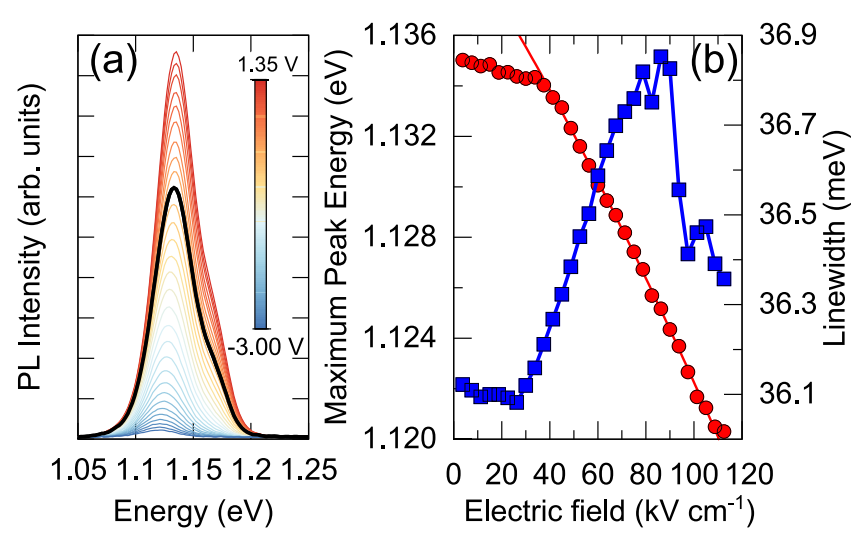

FIG. 2. (a) Evolution of the PL as a function of the external voltage. The black thick line corresponds to the $0 \mathrm{~V}$ spectrum. (b) Ground state peak energy (red dots) and FWHM (blue squares) are extracted from Gaussian deconvolution of the main peak. The solid line is the parabolic dependence predicted by the $\mathbf{k} \cdot \mathbf{p}$ model.
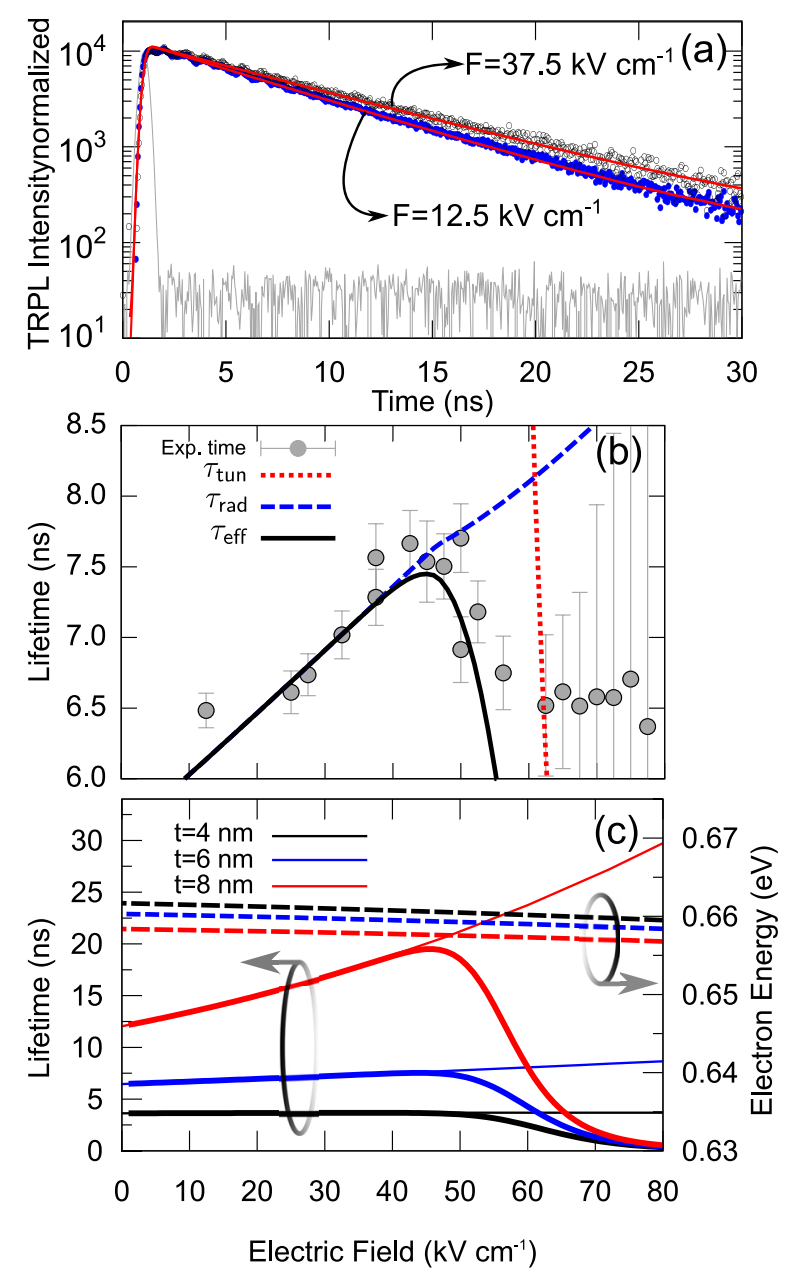

FIG. 3. (a) TRPL experimental data and decay time fits obtained at the indicated bias. The system response is plotted in grey. (b) Best fit of the theoretical radiative lifetime (dashed line), tunneling time (dotted line), and effective lifetime (thick solid line) to the experimental decay times (circles). (c) Bias evolution of the radiative lifetime (thin solid lines), effective lifetime (thick solid lines), and electron energy (dashed lines) calculated for different thicknesses of the GaAsSb QW.

$F W H M \approx 36 \mathrm{meV}$ and a high energy shoulder related to an excited state transition at $1.168 \mathrm{eV}(F W H M \approx 29 \mathrm{meV})$. The intensity of the main peak ranges from $79 \%$ of the total emission at $0 \mathrm{~V}$ to $94 \%$ at $-3 \mathrm{~V}$. Therefore, from now on we focus only on the fundamental transition. Figure 2(b) shows how from 40 to $110 \mathrm{kV} \mathrm{cm}^{-1}$ the experimental peak energy shifts by $13 \mathrm{meV}$, nearly a $40 \%$ of the inhomogeneous bandwidth, while the FWHM remains constant in the same range of electric fields (variation $<1 \mathrm{meV}$ ). ${ }^{28}$ Both the $\mathbf{k} \cdot \mathbf{p}$ method and perturbation theory curves produce a good fit to the data in this region. We find that the experimental value for $p / e=1.48 \mathrm{~nm}$ is in good agreement with the theoretical one $p / e=2.2 \mathrm{~nm}$ calculated for the nominal thickness of the GaAsSb QW, $t=6 \mathrm{~nm}$. The permanent dipole moment is related to the expectation value of the electron and hole $\mathrm{z}$ coordinate at $F=0 \mathrm{kV} \mathrm{cm}^{-1}$ through $p / e=\left\langle z_{\mathrm{e}-\mathrm{h}}\right\rangle=\left\langle z_{\mathrm{h}}\right\rangle-\left\langle z_{\mathrm{e}}\right\rangle$. The Coulomb interaction is expected to be small due to type II alignment, but even without it, our model predicts that the strain and piezoelectric potentials are enough to stabilize the hole wavefunction above the electron one at zero bias. In addition, we find that the polarizability $\beta_{\exp }=-0.32 \mu \mathrm{eV} \mathrm{kV}^{-2} \mathrm{~cm}^{2}$ also agrees 
well with the theoretical value $\beta_{\text {theo }}=-0.39 \mu \mathrm{eV} \mathrm{kV} \mathrm{cm}^{-2}$, concluding that the key parameters of the electrical response of these QDs are well reproduced by our model.

To analyze the carrier dynamics, we have performed time resolved PL (TRPL) experiments. Figure 3(a) contains two representative decay curves recorded at $5 \mathrm{~K}$ for two values of the electric field. The solid lines represent the convolution of the system response with the single exponential decay fits for each dataset. We obtain long decay times, in excess of $6 \mathrm{~ns}$, confirming that carriers exhibit type II confinement after the rapid thermal annealing treatment. ${ }^{29,30}$ The full evolution of the decay time with the external electric field is represented in Figure 3(b). For each point, the decay time and its statistical error were estimated at the maximum of the emission, following the Stark shift of the ground state. The decay time follows a curve typical of two counteracting processes. ${ }^{31}$ The region of lifetime increase $\left(F \lesssim 50 \mathrm{kV} \mathrm{cm}^{-1}\right)$ corresponds to the vertical separation of the electron and hole wavefunctions and the reduction of the electron-hole overlap dictated by the QCSE. The $\mathbf{k} \cdot \mathbf{p}$ predicts a change in the radiative lifetime $\tau_{\text {rad }}$ from 5 to $12 \mathrm{~ns}$ between $-150 \mathrm{kV} \mathrm{cm}^{-1}$ and $150 \mathrm{kV} \mathrm{cm}^{-1}$. The region of lifetime decrease $\left(F \gtrsim 50 \mathrm{kV} \mathrm{cm}^{-1}\right)$ shall be associated to the tunnelling of the electron through the InAs/ GaAs interface. The tunneling rate $\tau_{\text {tun }}^{-1}$ can be estimated using the Wentzel-Kramers-Brillouin approximation for a triangular well

$$
\frac{1}{\tau_{\text {tun }}}=\frac{\hbar}{8 m_{e}^{*} H^{2}} \exp \left\{-\frac{4}{3} \frac{\sqrt{2 m_{e}^{*}}}{e \hbar F}\left[E_{c}-E_{e}(F)\right]^{3 / 2}\right\},
$$

where we introduce the electron effective mass of compressed InAs, $m_{e}^{*}=0.1$; the GaAs band edge at the QD base, $E_{c}=0.765 \mathrm{eV} ; H=2.3 \mathrm{~nm}$, for the QD height determined independently; ${ }^{29}$ and $E_{e}(F)$ for the electron ground state energy given by the model. The resulting $\tau_{\text {tun }}$ is depicted by the dotted line in Figure 3(b). The effective lifetime is obtained as a sum of the rates $\tau^{-1}=\tau_{\text {tun }}^{-1}+\tau_{\text {rad }}^{-1}$. As displayed by the black solid curve in Figure 3(b), there is an optimum match with the experimental values by just rigidly downshifting the theoretical $\tau_{\text {rad }}$ (dashed line) by $1 \mathrm{~ns}$.

Large $p$ and $\beta$ are desirable to fabricate electroabsorption modulators with small modulation voltages and low insertion losses. Because of the larger polarizability of our QD structure, we expect a noticeable modulation of the oscillator strength and exciton radiative lifetime with the applied bias as described above. However, the tunneling of the electron out of the QD prevents to extend the tuning to higher electric fields. In this context, an additional feature arises from the large asymmetry between the electron and hole confinement, which allows to tune almost independently $\tau_{\text {rad }}$ and $\tau_{\text {tun }}$ in our system. Figure 3(c) shows how $\tau_{\text {rad }}$ and the effective lifetime scales up with the GaAsSb QW thickness and applied bias. This is a consequence of the large $\beta$ value of these QDs which is mostly due to the spatial modulation of the hole wavefunction in the GaAsSb layer. Meanwhile, the strongly confined electron does not change its energy with varying $F$, as depicted by the dashed lines in the same figure (less than $1 \mathrm{meV}$ for a given $t$ ). At the same time, due to their deeper confinement potential and large effective mass, holes do not tunnel through the GaAs/ $\mathrm{GaAsSb} \mathrm{QW}$ interface. Under this configuration, the tunneling of the electron through the InAs/GaAs interface determines entirely the exciton ionization and hardly depends on the particular GaAsSb layer thickness (only through small variations of the electron energy due to the strain field). Thus, both parameters can be tuned independently in a wide range, playing with the GaAsSb QW thickness to fix $\tau_{\text {rad }}$ and independently introducing lattice matched electron tunneling barriers to fix $\tau_{\text {tun }}$ as discussed in Ref. 32 .

The QCSE properties of the proposed structure arise not only from a modulation of the electron hole wavefunction overlap but also from a change of the hole wavefunction topology. An intuitive picture can be obtained from the probability density calculated by the eight-band $\mathbf{k} \cdot \mathbf{p}$ model as shown in Figure 4(a). For $F<0 \mathrm{kV} \mathrm{cm}^{-1}$, the combination of type II confinement, strain, and piezoelectric fields results in a hole wavefunction that adopts a non-single connected geometry (ring-like) and the formation of a large in-plane permanent dipole $\left\langle\rho_{\mathrm{e}-\mathrm{h}}\right\rangle$. Applying a magnetic field in the growth direction, both conditions shall induce a relative Berry phase between the electron and hole wavefunctions resulting in the periodic changes of certain optical magnitudes, such as the oscillator strength, and other effects predicted by Aharonov and Bohm (AB). ${ }^{33,34}$

These phenomena have received much attention in type I and type II semiconductor nanostructures connected to spin-orbit physics and applications. ${ }^{24}$ Type-I quantum rings $(\mathrm{QRs})$ with the correct confinement topology for electrons and holes are difficult to fabricate and result in rather small $\left\langle\rho_{e-h}\right\rangle$ (typically $\approx 2 \mathrm{~nm}$ ), making the detection of the optical $\mathrm{AB}$ effects a very challenging task. ${ }^{34,35}$ The application of a vertical electric field was then proposed to increase slightly $\left\langle\rho_{e-h}\right\rangle$ and identify tunable $\mathrm{AB}$ oscillations in the GaAs/AlGaAs QRs. ${ }^{36,37}$ In this regard, QDs with type II band alignment might be a better alternative to investigate the optical $\mathrm{AB}$ phenomena, ${ }^{38,39}$ although the possibility of a bias dependent topology has not been exploited in these systems yet.
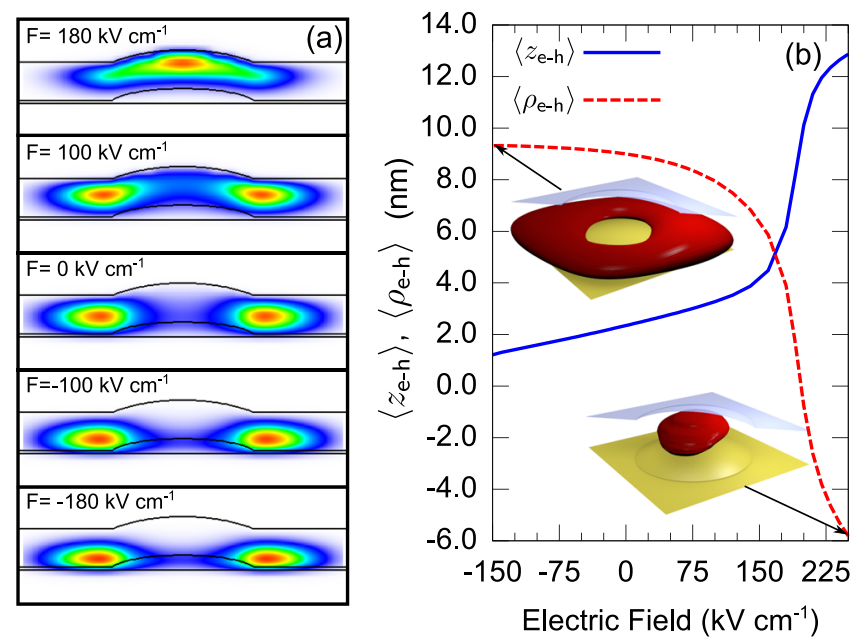

FIG. 4. (a) Probability density distribution of the fundamental state of holes averaged over (110) and (110) planes. (b) Expected value of vertical (blue solid line) and radial (red dashed line) separation between electron and hole. The insets show three dimensional colour plots of the ground state hole wavefunction probability density calculated at the indicated electric fields. 
Figure 4(b) shows the bias evolution of the vertical and in-plane dipole moments calculated for the $\operatorname{In}(\mathrm{Ga}) \mathrm{As} / \mathrm{GaAsSb}$ structure. In contrast to type I systems, at $F=0 \mathrm{kV} \mathrm{cm}^{-1}$, $\left\langle\rho_{\mathrm{e}-\mathrm{h}}\right\rangle$ is already $9 \mathrm{~nm}$ with $\left\langle z_{\mathrm{e}-\mathrm{h}}\right\rangle=2.3 \mathrm{~nm}$. Applying negative electric fields, the hole moves downwards approaching the electron and increasing the e-h overlap, as explained earlier. The in-plane dipole moment remains mostly unchanged in this bias region which would be most favorable for the observation of the optical $\mathrm{AB}$ effect. Reversing the field direction, the hole wavefunction starts to move upwards decreasing $\left\langle\rho_{\mathrm{e}-\mathrm{h}}\right\rangle$ down to zero at $F=200 \mathrm{kV} \mathrm{cm}^{-1}$. A sweep of the lateral dipole moment of this magnitude is unforeseen in the literature and shall pave the way for further investigations of the tunable optical $\mathrm{AB}$ effects in the semiconductor nanostructures.

In summary, we study the voltage control of the hole wavefunction geometry and topology in a type-II InAs/ GaAsSb dot-in-well structure. We demonstrated that the key parameters for semiconductor electro-optical applications in this structure can be tuned precisely in a wider range than using type-I QDs. We also predicted a bias regime where the lateral exciton dipole moment and the hole wavefunction topology enable the study of spin-orbit phenomena like voltage tunable optical Aharonov-Bohm effects.

The authors acknowledge the financial support from the Spanish MINECO through the Grant Nos. ENE201237804-C02-02, TEC2011-29120-C05-04, and MAT201347102-C2-2-R. Comunidad de Madrid through the Grant No. S2013/MAE-2780. We also thank SGAI-CSIC for the allocation of computational resources and Brazilian CNPq for supporting E.R.C. de Oliveira through the Grant No. 313812/2013-6.

${ }^{1}$ Ultrafast All-Optical Signal Processing Devices, edited by H. Ishikawa (John Wiley \& Sons Ltd., United Kingdom, 2008).

${ }^{2}$ C. J. Chang-Hasnain, P.-C. Ku, J. Kim, and S.-L. Chuang, Proc. IEEE 91, 1884 (2003).

${ }^{3}$ C.-Y. Jin and O. Wada, J. Phys. D: Appl. Phys. 47, 13300 (2014).

${ }^{4}$ O. Wada, IEEE J. Sel. Top. Quantum Electron. 17, 309 (2011).

${ }^{5}$ A. A. High, E. E. Novitskaya, L. V. Butov, M. Hanson, and A. C. Gossard, Science 321, 229 (2008).

${ }^{6}$ E. A. Stinaff, M. Scheibner, A. S. Bracker, I. V. Ponomarev, V. L. Korenev, M. E. Ware, M. F. Doty, and D. G. T. L. Reinecke, Science 311, 636 (2006)

${ }^{7}$ H. J. Krenner, C. E. Pryor, J. He, and P. M. Petroff, Nano Lett. 8, 1750 (2008).

${ }^{8}$ M. Kroutvar, Y. Ducommun, D. Heiss, M. Bichler, D. Schuh, G. Abstreiter, and J. J. Finley, Nature 432, 81 (2004).

${ }^{9}$ P. W. Fry, I. E. Itskevich, D. J. Mowbray, M. S. Skolnick, J. J. Finley, J. A. Barker, E. P. O’Reilly, L. R. Wilson, I. A. Larkin, P. A. Maksym, M. Hopkinson, M. Al-Khafaji, J. P. R. David, A. G. Cullis, G. Hill, and J. C. Clark, Phys. Rev. Lett. 84, 733 (2000).

${ }^{10}$ R. J. Warburton, C. Schulhauser, D. Haft, C. Schaflein, K. Karrai, J. M. Garcia, W. Schoenfeld, and P. M. Petroff, Phys. Rev. B 65, 113303 (2002).

${ }^{11}$ J. J. Finley, M. Sabathil, P. Vogl, G. Abstreiter, R. Oulton, A. I. Tartakovskii, D. J. Mowbray, M. S. Skolnick, S. L. Liew, A. G. Cullis, and M. Hopkinson, Phys. Rev. B 70, 201308 (2004).
${ }^{12}$ D. Alonso-Álvarez, B. Alén, J. M. García, and J. M. Ripalda, Appl. Phys. Lett. 91, 263103 (2007)

${ }^{13}$ K. L. Janssens, B. Partoens, and F. M. Peeters, Phys. Rev. B 67, 235325 (2003).

${ }^{14}$ See supplementary material at http://dx.doi.org/10.1063/1.4934841 for a detailed description of the fabrication, characterization and theoretical methods.

${ }^{15}$ A. G. Taboada, A. M. Sánchez, A. M. Beltrán, M. Bozkurt, D. AlonsoÁlvarez, B. Alén, A. Rivera, J. M. Ripalda, J. M. Llorens, J. MartínSánchez, Y. González, J. M. Ulloa, J. M. García, S. I. Molina, and P. M. Koenraad, Phys. Rev. B 82, 235316 (2010).

${ }^{16}$ A. G. Taboada, J. M. Llorens, D. Alonso-Álvarez, B. Alén, A. Rivera, Y. González, and J. M. Ripalda, Phys. Rev. B 88, 085308 (2013).

${ }^{17}$ J. M. Ripalda, D. Granados, Y. González, A. M. Sánchez, S. I. Molina, and J. M. García, Appl. Phys. Lett. 87, 202108 (2005).

${ }^{18}$ J. M. Ulloa, R. Gargallo-Caballero, M. Bozkurt, M. del Moral, A. Guzmán, P. M. Koenraad, and A. Hierro, Phys. Rev. B 81, 165305 (2010).

${ }^{19}$ J. M. Ulloa, J. M. Llorens, M. del Moral, M. Bozkurt, P. M. Koenraad, and A. Hierro, J. Appl. Phys. 112, 074311 (2012).

${ }^{20}$ P. Klenovský, V. Křápek, D. Munzar, and J. Humlíček, Appl. Phys. Lett. 97, 203107 (2010).

${ }^{21}$ N. Pavarelli, T. J. Ochalski, H. Y. Liu, K. Gradkowski, M. Schmidt, D. P. Williams, D. J. Mowbray, and G. Huyet, Appl. Phys. Lett. 101, 231109 (2012).

${ }^{22}$ A. Hospodková, M. Zíková, J. Pangrác, J. Oswald, J. Kubištová, K. Kuldová, P. Hazdra, and E. Hulicius, J. Phys. D: Appl. Phys. 46, 095103 (2013).

${ }^{23}$ K. L. Janssens, B. Partoens, and F. M. Peeters, Phys. Rev. B 65, 233301 (2002).

${ }^{24}$ Physics of Quantum Rings, edited by V. M. Fomin (Springer, Berlin Heidelberg, 2014).

${ }^{25}$ D. A. B. Miller, D. S. Chemla, T. C. Damer, A. C. Gossard, W. Weigmann, T. H. Wood, and C. A. Burrus, Phys. Rev. Lett. 53, 2173 (1984).

${ }^{26}$ S. Birner, T. Zibold, T. Andlauer, T. Kubis, M. Sabathil, A. Trellakis, and P. Vogl, IEEE Trans. Electron Devices 54, 2137 (2007).

${ }^{27}$ Electric field is approximated by the expression $F=-\left(V-E_{g, \mathrm{GaAs}}\right) / d$.

${ }^{28} \mathrm{Near}$ the flat band and up to $40 \mathrm{kV} \mathrm{cm}^{-1}$, a smaller slope was found. We attribute this effect to the screening of the external electric field by charged defects. We have observed that the screening is minimized under picosecond pulsed excitation with $10 \mathrm{MHz}$ repetition frequency suggesting that the traps have slower dynamics than the nanostructures.

${ }^{29}$ J. M. Ulloa, J. M. Llorens, B. Alén, D. F. Reyes, D. L. Sales, D. González, and A. Hierro, Appl. Phys. Lett. 101, 253112 (2012).

${ }^{30}$ W.-S. Liu, Y.-T. Wang, W.-Y. Qiu, and C. Fang, Appl. Phys. Express 6, 085001 (2013).

${ }^{31}$ B. Alén, J. Bosch, D. Granados, J. Martínez-Pastor, J. M. García, and L. González, Phys. Rev. B 75, 045319 (2007).

${ }^{32}$ A. J. Bennett, R. B. Patel, J. Skiba-Szymanska, C. A. Nicoll, I. Farrer, D. A. Ritchie, and A. J. Shields, Appl. Phys. Lett. 97, 031104 (2010).

${ }^{33}$ Y. Aharonov and D. Bohm, Phys. Rev. 115, 485 (1959).

${ }^{34}$ A. O. Govorov, S. E. Ulloa, K. Karrai, and R. J. Warburton, Phys. Rev. B 66, 081309R (2002).

${ }^{35}$ M. D. Teodoro, V. L. Campo, Jr., V. Lopez-Richard, E. Marega, Jr., G. E. Marques, Y. Galvão Gobato, F. Iikawa, M. J. S. P. Brasil, Z. Y. AbuWaar, V. G. Dorogan, Y. I. Mazur, M. Benamara, and G. J. Salamo, Phys. Rev. Lett. 104, 086401 (2010).

${ }^{36}$ F. Ding, N. Akopian, B. Li, U. Perinetti, A. Govorov, F. M. Peeters, C. C. Bof Bufon, C. Deneke, Y. H. Chen, A. Rastelli, O. G. Schmidt, and V. Zwiller, Phys. Rev. B 82, 075309 (2010).

${ }^{37} \mathrm{~B}$. Li and F. M. Peeters, Phys. Rev. B 83, 115448 (2011).

${ }^{38}$ E. Ribeiro, A. O. Govorov, W. Carvalho, and G. Medeiros-Ribeiro, Phys. Rev. Lett. 92, 126402 (2004).

${ }^{39}$ I. L. Kuskovsky, W. MacDonald, A. O. Govorov, L. Mourokh, X. Wei, M. C. Tamargo, M. Tadic, and F. M. Peeters, Phys. Rev. B 76, 035342 (2007). 